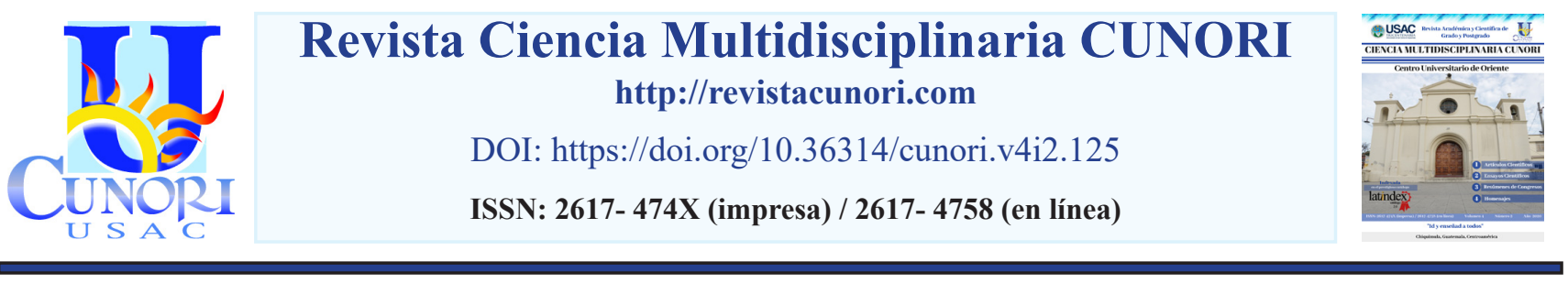

Referencia del artículo

Guerrero-Fragoso, D. (2020). Viviendo en la periferia: una visión desde la movilidad cotidiana. Revista Ciencia Multidisciplinaria Cunori, 4(2), 29-38. https://doi.org/10.36314/cunori.v4i2.125

\title{
Viviendo en la periferia: una visión desde la movilidad cotidiana
}

\author{
Living on the periphery: a vision form daily mobility
}

\author{
Diana Guerrero Fragoso \\ Doctorante del Programa de Posgrado de Geografía, Universidad Nacional Autónoma de México \\ https://orcid.org/0000-0001-8022-2356 \\ Recibido: 20 de enero de 2020 / Revisado: 15 de febrero de 2020 / Aceptado: 20 de mayo de 2020
}

Disponible en internet el 30 de octubre de 2020

*Autor para correspondencia.

Correo electrónico: geo.dianaguerrero@gmail.com

\section{Resumen}

$\mathrm{L}$ a movilidad cotidiana es uno de los fenómenos más relevantes para conocer las ciudades y sus dinámicas. El objetivo fue ana-

lizar los movimientos que tienen lugar en la periferia urbana de la Ciudad de México para conocer la importancia del ingreso y la localización en la forma de acceder y vivir la ciudad, así como rescatar las experiencias de viaje en el estudio de la movilidad para entender la dimensión social del fenómeno, y con ello sus implicaciones en la configuración socioespacial y en la dinámica en el territorio. Se desarrolló un análisis con metodología mixta, a través de encuestas y etnografías. Se estableció que mayores ingresos se asocian a una mayor movilidad y acceso a los servicios que ofrece la ciudad. También se concluye que la movilidad influye en la percepción de la calidad de vida de la población que habita en la periferia, pese a que el 63\% manifestó no ser consciente de la disponibilidad de tiempo libre durante la rutina semanal, así como del tiempo destinado a sus desplazamientos diarios.

Palabras clave: movilidad cotidiana, periferia urbana, experiencias de viaje, ingreso

Abstract

$\mathrm{D}$ aily mobility is one of the most relevant phenomenon to know cities and their dynamics. The objective was to analyze the movements that take place on the urban periphery of Mexico City to understand the importance of income and location in the way the city is accessed and lived, as well as to rescue travel experiences in the study of mobility to understand the social dimension of the phenomenon, and with it its implications in sociospatial configuration and dynamics in the territory. An analysis with mixed methodology was developed, through surveys and ethnographies. It was established that higher incomes are associated with greater mobility and access to the services offered by the city. It is also concluded that mobility influences the perception of the quality of life of the population living on the periphery, although $63 \%$ said they were unaware of the availability of free time during the weekly routine, as well as the time spent on their deplores daily displacements.

Keywords: daily mobility, urban periphery, travel experiences, incomes 


\section{Introducción}

Los desplazamientos cotidianos que tienen lugar en la Ciudad de México y su periferia urbana son fundamentales para definir el nivel de articulación territorial de la metrópoli más grande de México. Entender como es la relación funcional desde la perspectiva de la movilidad cotidiana, contribuye a explicar fenómenos, como el crecimiento de asentamientos humanos en territorios cada vez más alejados de la ciudad central y los principales núcleos urbanos que en términos de traslados implican distancias cada vez más grandes y por ende un incremento en los tiempos de viaje.

Sin embargo, el tiempo y la distancia son solo algunos de los aspectos más estudiados de la movilidad, las particularidades de los desplazamientos vistas a través de la experiencia individual del viaje permiten visibilizar las condiciones de los desplazamientos cotidianos y dar cuenta de que la movilidad cotidiana y el acceso a la ciudad está determinado por el nivel socioeconómico de la población, "la relación entre renta y movilidad, con una restricción de acceso a bienes y servicios, puede leerse como una limitación del derecho a la ciudad" (Miralles-Guasch, 2009:203)

En América Latina existen diversas aportaciones que analizan la relación de dependencia que existe movilidad y pobreza, la mayoría abordados desde la perspectiva de la accesibilidad y la exclusión social (Avellaneda, 2008; Jirón, 2009, Avellaneda y Lazo, 2011; Moreno y Rubiano, 2014; Hernández, 2018). Sin embargo, poco se ha profundizado en los matices de la movilidad cotidiana periurbana, partiendo desde la desigualdad y heterogeneidad socioespacial no solo en términos de la movilidad misma sino del acceso diferenciado y la experiencia urbana que está determinada por el ingreso.

Considerando que la movilidad se refiere al acto de desplazarse de un lugar a otro a través del uso de algún modo de transporte, pero también comprende el significado social y cultural de ese desplazamiento (Dávila, 2012) es que ha comenzado a reorientarse la discusión de la movilidad hacia la perspectiva de la construcción social.

Dado que la movilidad es una de las actividades que tiene mayor impacto en la vida del ser humano, pues dota a su cotidianeidad de múltiples experiencias e influye en su calidad de vida. Desde la sociología ha comenzado a analizarse la importancia de invertir poco tiempo en los traslados cotidianos al trabajo como parte fundamental de una buena calidad de vida. No obstante, en Latinoamérica se continúa privilegiando el uso del automóvil como un bien de consumo duradero, pese a que las cifras de congestionamiento vial van en aumento, de acuerdo con el Global Traffic Scorecard 2019 (Scorecard, 2019) Bogotá, Ciudad de México y Sao Paulo encabezaron la lista de las ciudades con peor tráfico vehicular a en América Latina y se encuentran dentro del ranking de las 10 Ciudades más cogestionadas del mundo.

De acuerdo con el Instituto Nacional de Estadística y Geografía (INEGI, 2018) el parque vehicular de la Ciudad de México creció en un 163\% en los últimos 25 años, lo que se ha traducido en un incremento en los tiempos de traslado, se estima que la velocidad promedio es de $6 \mathrm{~km}$ por hora, de acuerdo con la 
encuesta Origen-Destino 2017 y los tiempos de traslado en la ciudad aumentaron en 10\% en la última década. Sin embargo, el uso de automóvil particular mantiene su tendencia creciente con una tasa anual de $5.3 \%$ y existe poco interés por medir los efectos socioeconómicos y ambientales que tiene en la Ciudad de México.

En este sentido, el estudio de la movilidad ha promovido un importante debate sobre las metodologías empleadas, lo que ha permitido la búsqueda e incorporación de otras perspectivas que profundicen en las especificidades de las movilidades. Este articulo hace énfasis en el uso de metodología cualitativa para dar cuenta de las experiencias de quienes se desplazan y no sólo de los modos de transporte.

Los casos de estudio corresponden al denominado corredor Norponiente de la Ciudad de México, ubicado en la periferia urbana de la ciudad, que destaca por ser una zona con gran heterogeneidad socioespacial, además de ser la entidad con mayor crecimiento en su parque vehicular con 1.5 millones de unidades (INEGI, 2018) y que contrasta con insuficiente infraestructura vial.

\section{Materiales y métodos}

Se empleó una metodología mixta, incorporando técnicas cuantitativas y cualitativas, consistente en la aplicación de 120 encuestas y la realización de cuatro etnografías. La combinación de ambas técnicas permite un análisis más profundo del fenómeno a investigar ¿cómo y cuál es el propósito del viaje?, ¿qué sucede en su transcurso?, ¿cuáles son los efectos de estos viajes en términos culturales, económicos y sociales?, ¿qué tipo de espacialidad surge a partir de las prácticas de movilidad?, son algunos aspectos que permiten entender la forma en la que el ingreso determina la manera en que se accede y se vive la ciudad.

En cuanto al método cualitativo, fue empleada la etnografía de tipo multisituada, basada en dar seguimiento al movimiento de personas, para dar cuenta de objetos, prácticas y discursos, concretamente a través de la técnica del sombreo, propuesta por Jirón en 2010, que consiste en una modalidad de acompañamiento a los informantes, en sus viajes y rutinas diarias.

Teniendo en cuenta el enfoque de ingreso como aspecto que condiciona y determina la movilidad cotidiana, se exponen cuatro casos de estudio correspondientes a nivel bajo, medio (dos casos) y alto, representativos de tres de los cuatro municipios que integran la zona de estudio, la selección se realizó con base en su localización geográfica dentro del denominado corredor Norponiente de la Ciudad de México, el corredor Norponiente de la Ciudad de México está integrado por los municipios de Nicolás Romero, Atizapán de Zaragoza, Tlalnepantla y Naucalpan, zona de estudio de la tesis doctoral de la autora.

En el presente estudio se muestran las etnografías realizadas en tres de los cuatro municipios, elegidas por su representatividad del tema., así como la particularidad de sus desplazamientos. Cada persona 
participante en el estudio fue considerada un caso. Se establecieron tres categorías dadas por su localización y nivel socioeconómico: Bajo, medio y alto. A partir del acercamiento a través de la encuesta fueron seleccionados los informantes clave para la técnica del sombreo.

\section{Resultados}

La encuesta se estructuró con 25 preguntas, los principales resultados se muestran a continuación: la distribución por sexo de la muestra fue de 68 hombres (57\% de la muestra) y 52 mujeres (47\% de la muestra). E1 34\% de los entrevistados se concentró en el grupo de edad de 31 a 45 años, seguido con el $28 \%$ en el rango de 46 a 60 años, el de $46-59$ años con $23 \%$ y por último mayores de 60 años con $15 \%$.

\section{Las ocupaciones de los informantes}

Todos los individuos de la muestra son residentes del Estado de México, en cuanto a sus ocupaciones, el $61 \%$ es población que trabaja (clasificados en el rubro de empleados $39 \%$ y $22 \%$ trabajadores por cuenta propia) el 16\% estudiantes, $15 \%$ amas de casa, $8 \%$ jubilados y $1 \%$ sin ocupación.

\section{El ingreso que perciben}

En la pregunta de ingreso, el 25\% percibe de 2 a 3 salarios, en la misma proporción del grupo que no percibe ingresos, el 19\% tiene ingresos entre 1 y 2 salarios mínimos, el 10\% percibe hasta un salario mínimo, 9\% más de 5 salarios y $8 \%$ entre 3 y 5 salarios mínimo.

\section{Uso de transporte público o privado}

En relación a la frecuencia del uso de transporte público o privado el 59\% afirma utilizarlo entre 5-6 días a la semana, seguido por el grupo de población que lo emplea todos los días con un $28 \%$, en igual proporción con $3 \%$ quienes no lo utilizan o lo hacen entre 1 y 2 días por semana. El modo de transporte más empleado para la realización de sus actividades cotidianas es el público con $51 \%$, seguido por el automóvil con $37 \%$, bicicleta con $6 \%$, caminar $4 \%$ y taxi $3 \%$.

\section{Modo de transporte empleado para realizar sus actividades cotidianas}

Considerando la multimodalidad de los viajes cotidianos se preguntó cuántos modos de transporte emplea diariamente para realizar sus actividades, los resultados señalan que el 34\% de la población emplea dos modos de transporte, seguido con el $33 \%$ quien emplea 1 y con $29 \%$ quienes emplean 3 modos de transporte.

\section{Motivo principal de los desplazamientos cotidianos}

Los motivos principales de los traslados cotidianos corresponden en 57\% a trabajar, 14\% estudiar, $13 \%$ ir de compras, $9 \%$ ocio, $4 \%$ realizar algún trámite, $3 \%$ atención médica y $1 \%$ otro motivo. 


\section{Horario de inicio habitual de sus actividades}

El horario habitual en el que viajan para iniciar sus actividades diarias es matutino, la mayor proporción se registra en el rango de 7 a 9 am con $33 \%$, seguido por el $26 \%$ de 5 a $7,16 \%$ de 9 a 11 y $24 \%$ distribuido en el horario vespertino.

\section{Duración promedio de los viajes diarios}

La duración promedio de los viajes se distribuye con el 29\% que afirman viajar entre 61-90 minutos, $20 \%$ en la categoría de 31-60 minutos y en la de 91-120 minutos.

\section{El costo del transporte diario}

En relación al costo del transporte diario el 35\% gasta entre 21 a 50 pesos, el 33\% gasta entre $\$ 51$ y 75 pesos, $11 \%$ en el rango de $\$ 76-\$ 100$.

\section{Percepción del servicio del transporte público}

Se formularon dos preguntas para conocer la percepción en cuanto al servicio de transporte público, en términos de calidad y problemas, el $40 \%$ considera que el servicio es regular, el $33 \%$ afirma que es malo, el $16 \%$ que es bueno y el $9 \%$ pésimo. En cuanto a los principales problemas el $35 \%$ señalo la inseguridad en las unidades, el $24 \%$ refieren un mal servicio, $17 \%$ las malas condiciones de las unidades, el $15 \%$ el costo de la tarifa y el $8 \%$ la saturación de las unidades. Se les pregunto sí consideraban el tiempo invertido en sus traslados como un problema del transporte y el 97\% sostuvo que sí, en la pregunta asociada al tiempo invertido y la calidad de vida el $84 \%$ considero que el tiempo que pasan en transportarse sí influye en su calidad de vida. Cuatro preguntas fueron focalizadas de acuerdo al tipo de transporte empleado. A los usuarios de transporte particular se les preguntó cuántas personas en promedio viajaban con ellos en una semana de actividades laborales, el $68 \%$ viaja solo, $18 \%$ con una persona, $11 \%$ con 2 personas y $2 \%$ con más de 2 personas. La segunda pregunta fue sí considerarían ser usuarios del transporte público sí ofreciera un mejor servicio, el 61\% afirma que sí, mientras que el 34\% no lo considera. En tanto que, a los usuarios de transporte público se les pregunto sí estarían interesados en adquirir un vehículo o usarían uno sí estuviera dentro de sus posibilidades, el 89\% respondió que sí, el $8 \%$ no; al cuestionar el motivo el $37 \%$ afirma que por seguridad. El 30\% por comodidad, $25 \%$ rapidez y un $5 \%$ considera que es más barato.

\section{Etnografías realizadas}

En relación a las etnografías realizadas con el uso de la técnica de sombreo, se presentan las particularidades de cuatro casos de estudio:

\section{La jornada de Andrés}

Andrés tiene 48 años, está casado y tiene 2 hijas, ha vivido en la colonia Lomas de Valle Escondido los últimos 12 años, un fraccionamiento exclusivo ubicado al poniente del municipio de Atizapán de 
Zaragoza, el acceso es controlado con seguridad privada. Es Ingeniero en Sistemas Computacionales y trabaja desde 2012 en el Corporativo de Santander ubicado en Santa Fe.

Su rutina inicia a las 6:00 am, se ejercita, desayuna y sale de su casa alrededor de las 7:30 viaja solo en su automóvil, toma la autopista Chamapa-Lechería en la entrada Madín. Su trayecto dura en promedio 40 minutos, cuando el tiempo se lo permite suele pasar por un café y generalmente llega con minutos de sobra a su trabajo, aunque no tiene una hora de entrada le gusta llegar antes de las 9am. En su hora de la comida a las 2pm, generalmente sale a los lugares más cercanos donde le gusta llegar caminando o bien sale con sus compañeros, compartiendo un solo auto, porque afirma que no se puede caminar en Santa Fe. Generalmente sale de su trabajo a las 5:30, en el trayecto de regreso su esposa quien le pidió que recogiera a su hija menor quien saldría de su clase de jazz, esto muy cerca de su casa (de paso sobre la ruta), a las 6:45 estábamos llegando a su casa. El resto de su día refiere pasarlo en casa con su familia.

\section{La jornada de Mariel}

Mariel es dentista y está por obtener su grado, estudio en la FES Iztacala y desde enero de 2018 está trabajando como asistente en un consultorio ubicado en la colonia Huichapan en la delegación Miguel Hidalgo, a un par de calles del Metro Tacuba. Tiene 23 años, es soltera y no tiene hijos, vive con sus papás y un hermano en la colonia San Lucas Patoni en Tlalnepantla, prácticamente en los límites con la Ciudad de México y muy cerca del reclusorio Norte, ahí ha vivido toda su vida, la zona es de clase media, aunque comenta que desde hace algunos años ha comenzado a ser más notorio un ambiente de inseguridad y delincuencia.

Su día comienza a las 7am, se baña y arregla para salir, prepara su comida y algo para desayunar en el camino, realiza un trayecto de 35 minutos, al metro La Raza, el trayecto en el metro dura 55 minutos con muchas complicaciones para abordar los trenes, con prisa, incertidumbre por la lentitud y los retrasos en el paso del metro, entre apretujones y empujones viaja toda la semana. Desde que entró a trabajar ahí siempre ha comido en el consultorio, pues siente un poco de inseguridad por la zona, esto mismo la ha obligado a esperar a que su jefa termine de atender a su último paciente, a veces hasta las 8pm, para que le dé un "aventón" al metro, o bien toma un taxi, ese día optamos por tomar un taxi, pero esta vez hacia el metro Panteones que es más seguro (porque no está el mercado y no hay que caminar tanto). Llega a su casa a las 8:50 pm, generalmente a unos 5 minutos de la parada de su casa llama a su papá para que salga por ella, el día del viaje se sintió acompañada y no lo hizo. Al llegar a su casa sólo cena y duerme, la mayoría de las veces sus padres están esperándola para cenar y ver TV.

\section{La jornada de José Roberto}

José Roberto es originario de Atizapán, tiene 35 años está casado y tiene 1 hijo, vive en la colonia Profesor Cristóbal Higuera, sólo conocida como "La Higuera" vive con su esposa e hijo y sus suegros desde hace 3 años cuando se casó, antes de casarse vivió en casa de sus padres en la colonia El Potrero a unos 20 minutos aproximadamente de su domicilio actual. 
Desde hace 1 año trabaja en CONAGUA, aproximadamente 8 meses antes entrar a trabajar estuvo desempleado y decidió comprar un auto y ser chofer de UBER, cuando entro a trabajar consideró que le resultaba más barato llegar a su trabajo en transporte público, además de evitarse el problema de buscar estacionamiento. Sin embargo, después de experimentar viajes promedio de 2.30 minutos decidió que ese tiempo perdido podía aprovecharlo trabajando extra, en lugar de pasarlo en el tráfico sin ningún beneficio adicional y por el contrario mayores gastos. Así que decidió regresar a trabajar su auto en UBER, antes y después de su empleo y desde entonces así lo ha hecho.

Para José Roberto, un día normal empieza a las 4:30am, sale de su casa a las 5:00am, se conecta (a Uber) cuando ya sale de su colonia, no le gusta aceptar viajes por ahí porque las colonias son inseguras, tiene días donde opta por ciertas rutas pues ya tiene conocimiento de cómo "se mueve la gente" por ejemplo, los lunes sale un poco más tarde (5:30) y le gusta ir hacia Chiluca y Valle Escondido, pues son comunes los viajes al aeropuerto y generalmente "le toca" tarifa dinámica, por lo que un viaje al aeropuerto sale alrededor de los $\$ 500$, en promedio hace 3 viajes, sí tiene suerte 5 viajes antes de llegar a su trabajo a las 9:00am, dice que la mayoría lo dejan en la zona centro o sur de la ciudad y con la opción que da Uber para "direccionarse" hacia algún punto generalmente tiene suerte y logra hacer su último viaje muy cercano a su trabajo.

A las 6:00pm, sale de su trabajo y dependiendo de su cansancio opta por direccionarse desde su salida hacia su domicilio, haciendo un par de viajes, aunque en esos casos han sido pocas las ocasiones de que algún pasajero viaje a una dirección muy próxima a su domicilio, en el resto de las ocasiones trabaja en promedio hasta las 9pm (pues a esa hora disminuye el tráfico). Los fines de semana dependiendo de su estado de ánimo y cansancio suele trabajar medio día, pues según él le gusta manejar cuando la ciudad está tranquila.

\section{La jornada de Cecilia}

Cecilia tiene 37 años es madre soltera de 3 hijos. Ella renta un cuarto en una vecindad ubicada en la colonia El molinito en Naucalpan, ha vivido en esa colonia desde hace 2 años, aunque en diferentes domicilios.

Tiene 3 años trabajando en un restaurante ubicado en Echegaray en Naucalpan. El día de Cecilia empieza a las 5:00 am, su horario de entrada es a las 6:00am, camina unos 7 minutos a la avenida donde toma el transporte público que la deja en el mercado de San Bartolo, a esa hora ya circula mucho transporte, ahí toma otra combi que la deja a 3 calles de su trabajo, poco menos de 10 minutos.

Sale de su trabajo a las 6 pm, toma nuevamente una combi al mercado de San Bartolo donde casi todos los días pasa a comprar algo, ahí mismo toma la combi que la deja cerca de su casa, debe caminar unas 7 cuadras para pasar a recoger a sus hijos con una prima que le hace favor de cuidarlos. 


\section{Discusión}

En toda ciudad trasladarse es una actividad inherente al ser humano, pero hacerlo fácil y eficazmente es un valor agregado en el cumplir de las actividades cotidianas, la calidad de vida está sometida no solo a la posesión de bienes materiales sino también de las experiencias vividas.

La población del corredor Norponiente de la Ciudad de México, en su mayoría usuaria del transporte público tiene patrones de desplazamiento característicos de la periferia urbana, uno de ellos es la multimodalidad, el desplazamiento en grandes distancias y el tiempo invertido en ello.

En términos de costos económicos y sociales, el 80\% de la población destina más de $\$ 50$ pesos en trasladarse diariamente, el equivalente a medio salario mínimo. En cuanto al costo social de la movilidad, el 76\% de la población realiza desplazamientos de más de 30 minutos y califican su experiencia en el transporte público como regular en $40 \%$ y mala en 33\%, siendo la inseguridad el principal problema que los usuarios identifican.

Al cuestionar a los usuarios del transporte por la posibilidad de ser usuarios de transporte particular el 89\% manifestó estar interesados, siendo la seguridad, comodidad y velocidad las que identifican como principales ventajas de usar automóvil. Mientras que a los automovilistas se les cuestiono sobre el número de acompañantes que viajan comúnmente con ellos, el 68\% viaja solo, también se les cuestiono la posibilidad de usar el transporte público sí este ofreciera un buen servicio, el 61\% contesto que sí.

Un hallazgo importante es que los usuarios identifican el tiempo que les toma trasladarse, pero no consideraron el tiempo invertido como un problema del transporte, al replantear la pregunta asociándolo a la calidad de vida, el 97\% afirmo que el tiempo que invierten en sus viajes influye en su calidad de vida.

Este aspecto se abordó a profundidad con los cuatro participantes de la técnica de sombreo. Andrés de nivel socioeconómico alto, dispone de tiempo libre para realizar otras actividades antes y después de su jornada laboral en contraste con los otros tres casos, donde Mariel y José Roberto no disponen de tiempo para otras actividades y su convivencia familiar se limita a los fines de semana. José Roberto sostuvo que son pocas las ocasiones en que logra ver a su esposa al regresar del trabajo. En el caso de Cecilia la situación es similar, pero además su movilidad es limitada y muchas decisiones de consumo y empleo están determinadas por el gasto que puede destinar para el transporte.

En términos de transporte Andrés y José Roberto se mueven en automóvil todos los días, pero los ingresos de ambos están separados por una brecha superior a 4 salarios mínimos, Andrés manifiesta tener un buen acceso a la ciudad y la ubicación de su casa y empleo permite satisfacer sus necesidades de consumo fácilmente, la posibilidad de pagar por vialidades menos congestionadas es una ventaja sobre los automovilistas promedio que circulan por rutas más saturadas. Mientras que José Roberto ha implementado tener un doble empleo como estrategia al trafico cotidiano que vive entre su domicilio y su trabajo. 
En este estudio se ha abordado como la acción de transportarse es hoy en día un aspecto fundamental en la vida cotidiana de los habitantes del corredor Norponiente. Fueron expuestas algunas percepciones que tienen los usuarios sobre su transporte diario y las experiencias que caracterizan y dan forma su movilidad, visibilizando otra perspectiva del fenómeno.

\section{Agradecimientos}

La realización del trabajo de campo fue posible gracias al apoyo de la Universidad Nacional Autónoma de México, a través del Programa de Apoyo a Estudios de Posgrado (PAEP) en la partida 215.

\section{Referencias}

Avellaneda, P. (2008). Movilidad cotidiana, pobreza y exclusión social en la Ciudad de Lima. Anales de Geografía, 28 (2), 9-35.

Avellaneda, P. y Lazo, A. (2011). Aproximación a la movilidad cotidiana en la periferia de dos ciudades latinoamericanas. Los casois de Lima y Santiago de Chile. Revista Transporte y Territorio (4), 4758. https://doi.org/10.34096/rtt.i4.256

Dávila, J. (2012). Movilidad urbana y pobreza. Aprendizajes de Medellín y Soacha, Colombia. Medellin: The Development Plannin Unit, UCL.

Hernández, J. (2018). Influencia de la estructura urbana en los patrones de movilidad cotidiana de un trabajo feminizado en la Zona Metropolitana de la Ciudad de México. Perspectiva Geográfica 23 (2), 127-147. https://doi.org/10.19053/01233769.7380

INEGI. (2018) Encuesta Origen Destino 2017. Obtenido de https://www.inegi.org.mx/programas/ $\operatorname{eod} / 2017 /$

Miralles-Guasch, C. y. Cebollada. A. (2009). Movilidad cotidiana y sostenibilidad, una interpretación desde la geografía humana. Boletín de la A.G.E.. No. 50, 193-216. https://doi:10.1016/j. jtrangeo.2008.07.009

Moreno, C. y Rubiano M. (2014). Segregación residencfial y movilidad cotidiana en el contexto metropolitano. Un estudio a partir de las relaciones Bogotá-Soacha. Revista Territorios (31), 133-162. http:// dx.doi.org/10.12804/territ31.2014.06

Scorecard, G. T. (23 de 11 de 2019). Inrix. Obtenido de https://inrix.com/scorecard/

\section{Sobre la autora}

\section{Diana Guerro Fragoso es}

Geógrafa y Maestra en Urbanismo por la Universidad Nacional Autónoma de México, actualmente doctorante del Programa de Posgrado en Geografía. Su línea de investigación es la Geografía Urbana, el estudio de la desigualdad socioespacial y las dinámicas urbanas, desde la perspectiva de la movilidad. 


\section{Copyright (c) Diana Guerrero Fragoso}

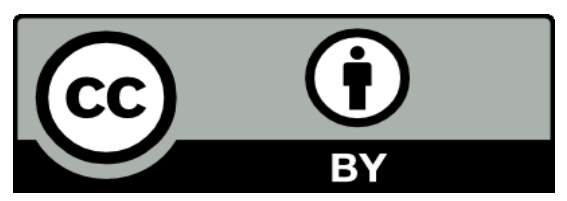

Este texto está protegido por una licencia CreativeCommons 4.0.

Usted es libre para compartir, copiar y redistribuir el material en cualquier medio o formato y adaptar el documento, remezclar, transformar y crear a partir del material para cualquier propósito, incluso comercialmente, siempre que cumpla la condición de atribución: usted debe reconocer el crédito de una obra de manera adecuada, proporcionar un enlace a la licencia, e indicar si se han realizado cambios. Puede hacerlo en cualquier forma razonable, pero no de forma tal que sugiera que tiene el apoyo del licenciante o lo recibe por el uso que hace. 